\title{
Spectroscopy of possible $\mathrm{H} \alpha$ emission stars in regions of high galactic latitude molecular clouds ${ }^{\star}$
}

\author{
E.L. Martín ${ }^{1}$ and M. Kun ${ }^{2}$ \\ 1 Instituto de Astrofísica de Canarias, 38200 La Laguna, Tenerife, Spain \\ 2 Konkoly Observatory, P.O. Box 67, H-1525 Budapest, Hungary
}

Received January 26; accepted October 9, 1995

\begin{abstract}
We present mid-resolution spectroscopic observations of 63 faint $(V=12-16)$ stars identified by Kun (1992) as $\mathrm{H} \alpha$ emission candidates in an objective prism survey of several high galactic latitude molecular clouds. Only 4 stars in our sample $(6 \%)$ are bona fide T Tauri stars on the basis of their strong Li I absorption and H $\alpha$ emission features. They have late M spectral types (M4-M5.5) and two of them form a visual binary with separation $\sim 7^{\prime \prime}$. The new T Tauris are associated with the L 134 molecular complex, the northernmost extension of the Scorpio Ophiuchus star forming region, which has been traditionally considered as a non-star forming region. In the other high latitude clouds surveyed by Kun (1992), we have not found any T Tauri star in our follow-up spectroscopy. Most of the observed stars $(\sim 80 \%)$ are late type dwarfs without detectable $\mathrm{H} \alpha$ emission in our spectra. Eight stars are M-dwarfs with $\mathrm{H} \alpha$ in emission, but no detectable Li I $\lambda 6708$ absorption. They could be post T Tauri stars or older dMe stars; more data is necessary in order to establish their evolutionary status. Our results show that Kun (1992) was able to detect weak $\mathrm{H} \alpha$ emission lines (down to equivalent width of $\sim 1.5 \AA$ ) in faint stars near the plate magnitude limit $(V \sim 16)$, but in many cases plate defects, absorption bands and/or overlying stars were taken as possible H $\alpha$ emission. In this paper we correct Kun's previous indication that there may be numerous young stars associated to high latitude molecular clouds, and we severely constrain the presence of a population of T Tauri stars in these clouds. We note that none of our four $\mathrm{T}$ Tauri stars is located in an isolated translucent molecular cloud.
\end{abstract}

Key words: stars: pre-main sequence — stars: emission line — surveys

\section{Introduction}

Since the discovery of molecular clouds at high galactic latitudes (Magnani et al. 1985) considerable effort has been devoted in order to reveal their star formation activity. Surveys for young stellar objects (YSOs) have used the most conspicuous features of low mass young stellar objects: $\mathrm{H} \alpha$ emission, far infrared radiation, and X-ray emission. Kun (1992) reported on the discovery of $100 \mathrm{H} \alpha$ emission candidates during an objective prism survey performed with the Schmidt telescope of Konkoly Observatory. More recently, Magnani et al. (1995) provided a list of IRAS Faint Source Catalog objects in high galactic latitude fields having infrared signatures characteristic of young stellar objects. Both types of objects can be re-

Send offprint requests to: E.L. Martín

* Based on observations made with the Isaac Newton telescope, operated on the island of La Palma by the Royal Greenwich Observatory in the Spanish Observatorio del Roque de los Muchachos of the Instituto de Astrofísica de Canarias garded as candidate YSOs which need spectroscopic identification.

Follow-up observations of YSO candidates to date have not supported the occurrence of low-mass star formation in translucent clouds. Magnani et al. (1990) performed CCD imaging observations of a sample of IRAS point sources in 17 translucent cloud fields but found no premain sequence (PMS) stars among them. Caillault et al. (1995) surveyed the Einstein database for candidate PMS stars. They could only find one high-latitude PMS star located in a dark cloud. A few other T Tauri stars and PMS stars have been identified in high latitude clouds (e.g. Magnani et al. 1995, and references therein), but so far no translucent cloud has been proved to form low mass stars.

$\mathrm{H} \alpha$ emission is the most conspicuous optical feature of $\mathrm{T}$ Tauri stars. Objective prism $\mathrm{H} \alpha$ surveys are therefore widely used for finding candidate pre-main sequence stars. Detection of $\mathrm{H} \alpha$ emission is, however, insufficient criterion for establishing the PMS nature of a star. Moreover, very low dispersion objective prism spectra do not make the 
detection of this feature possible with certainty (see e.g. Ogura \& Sato 1990). For instance, the shape of the continuum of M-type stars near the magnitude limit of the photographic plate can mimic an emission line. Occasionally, plate defects or two overlapping spectra may show up as an emission line. On the other hand, the emission may be variable in time (e.g. Balázs et al. 1987). Therefore objective prism $\mathrm{H} \alpha$ surveys do not provide a reliable picture of the PMS population. Spectroscopic follow-up of $\mathrm{H} \alpha$ emission stars found during objective prism surveys is needed to establish the real nature of these objects.

First, the reality of the emission line has to be proved, then further criteria are needed in order to distinguish YSOs from dMe stars and other non-PMS $\mathrm{H} \alpha$ emission objects. Presence of a strong Li I $\lambda 6708$ absorption line is a reliable indicator of young age for late type PMS stars because they deplete Li very efficiently (Martín et al. 1994a). Strong Li depletion in M-type stars has been reported among evolved weak T Tauris (Martín et al. 1994a), post T Tauris (Martín \& Brandner 1995), and young cluster members (cf. $\alpha$ Per, Zapatero-Osorio et al. 1995). Hence, detection of Li in an M-type star is a very good indication of $\mathrm{T}$ Tauri status. For stars which have depleted their $\mathrm{Li}$, it is more complicated to establish their evolutionary status. Accurate distances and photometry are needed to place them in the HR diagram and compare with theoretical isochrones.

In this paper, we will focus on detecting $\mathrm{H} \alpha$ emission and Li I $\lambda 6708$ absorption as primary criteria for PMS status in the objects identified by Kun (1992). Stars with both $\mathrm{H} \alpha$ emission and Li I absorption trace recent sites of star formation, while stars with only $\mathrm{H} \alpha$ emission but no detectable Li, may trace older star formation events. The PMS nature of the latter stars will have to be established in future works.

\section{Spectroscopy}

Spectroscopic observations were conducted at the $2.5 \mathrm{~m}$ Isaac Newton Telescope with the Intermediate Dispersion Spectrograph during 5 nights in April 1993 and 3 nights in June 1994. Additionally, six stars were observed during a service night on July 15, 1994. All 29 stars which Kun (1992) quoted as possibly having strong or very strong $\mathrm{H} \alpha$ emission, were observed. In addition we selected more than half of the remaining stars, giving a total sample of 63 stars. We used the $235 \mathrm{~mm}$ camera and the 600 grooves grating, which provided a nominal dispersion of $1.58 \AA \mathrm{pix}^{-1}$. The slit width was 1.2 arcsec, which matched well the seeing conditions. The effective resolution obtained was $\mathrm{FWHM}=2.8 \AA$, and the spectral range $\lambda \lambda 5725-7380$.

The exposure times ranged from 300 to $1200 \mathrm{~s}$, depending on the apparent visual magnitude on the TV acquisition screen. The $S / N$ ratio obtained was always higher than 10 , and usually higher than 30 . Inspection of the raw data at the telescope was sufficient to detect $\mathrm{H} \alpha$ emission stronger than $\sim 1 \AA$. If an emission line was seen, the star was reobserved in the same night. In fact, after careful data reduction and analysis we could not detect any $\mathrm{H} \alpha$ emission line which we had not noticed at the telescope.

The reduction was done using $\mathrm{IRAF}^{1}$ tasks. Overscan correction and flat fielding were applied. We checked that the flat field exposures (taken from a tungsten lamp) did not have any spectral line. Optimal extraction and wavelength correction using arc lamps completed the reduction procedure. The rms of the third order polynomial dispersion solutions were lower than $0.05 \AA$.

\section{Results}

Most of the observed stars did not show $\mathrm{H} \alpha$ emission. In fact, many had $\mathrm{H} \alpha$ in absorption. We distinguish the following cases: i) $\mathrm{G}$ and K-type stars with $\mathrm{H} \alpha$ seen in absorption or undetected. ii) M-type stars with no detected $\mathrm{H} \alpha$ emission. iii) Stars with detected $\mathrm{H} \alpha$ emission. The stars that fall in category i) have the following Kun's (1992) numbers: 6, 10, 13, 14, 18, 19, 20, 23, 24, 25, $26,27,28,30,31,34,41,44,47,56,57,61,68,71,72,75$, $79,80,87,89,93,95,98$, and 99 . There are 34 stars in this class, which represents just over $50 \%$ of the sample. Checking the appearance of these stars on the objective prism plates in view of the spectroscopic results, we find that plate defaults, confusion with nearby stars, and the presence of absorption bands in stars later than K5, might explain the misclassification in most cases. In some cases, however, we do not find a satisfactory explanation why the appearance of these stars suggests the presence of an emission line. One unlikely possibility may be a real temporary emission due to sporadic stellar activity. Object number 21 turned out to be a catalogued Seyfert Galaxy.

Spectral types later than K5 can be estimated using the depth of molecular band heads. Since all the stars where we have detected $\mathrm{H} \alpha$ emission are late-K or Mtype, we have made a careful spectral classification of these cool stars. Several spectral ratios have been used in the literature for classifying M-stars, but none of them is based on spectra of the same resolution and coverage as ours. Hence, we decided to observe several spectral standards with the same setup as our programme stars, and define in them ratios sensitive to temperature and gravity.

We selected standards from Kirkpatrick et al. (1991), and we measured in our spectra the features listed in Table 1 . The I1 index is the same as the A index defined by Kirkpatrick et al.

Additionally, we also measured the equivalent width of the NaI resonance doublet at $\lambda 5890-5896$, as an indicator

\footnotetext{
${ }^{1}$ IRAF is distributed by National Optical Astronomy Observatory, which is operated by the Association of Universities for Research in Astronomy, Inc., under contract with the National Science Foundation.
} 
Table 1. Spectral ratios of cool stars

\begin{tabular}{llll}
\hline Name & Numerator & Denominator & Feature measured \\
\hline I1 & $7020-7050 \AA$ & $6960-6990 \AA$ & $\mathrm{CaH}$ \\
$\mathrm{I} 2$ & $6510-6540 \AA$ & $6370-6400 \AA$ & $\mathrm{CaH}$ \\
$\mathrm{I} 3$ & $6510-6540 \AA$ & $6660-6690 \AA$ & $\mathrm{TiO}$ \\
\hline
\end{tabular}

of gravity. In Table 2, we present the measured values in a set of dwarfs and giants. Note that the three indices defined in Table 1 increase monotonically from K5V to M6V. The indices measuring the strength of the $\mathrm{CaH}$ molecule are weak in giants, and the NaI equivalent width is systematically weaker in giants than in dwarfs.

Table 2. Standards

\begin{tabular}{llllll}
\hline Star & Sp.T. & I1 & I2 & I3 & EW (NaI) \\
\hline HR 8085A & K5V & 1.03 & 1.05 & 1.02 & 8.0 \\
HR 8085B & K7V & 1.07 & 1.12 & 1.06 & 9.1 \\
GJ 338A & M0V & 1.10 & 1.17 & 1.09 & 10.6 \\
GJ 338B & M0V & 1.11 & 1.21 & 1.04 & 10.0 \\
GJ 767A & M1V & 1.17 & 1.32 & 1.17 & 11.1 \\
GJ 767B & M2.5V & 1.23 & 1.41 & 1.35 & 8.8 \\
GJ 569A & M3V & 1.24 & 1.42 & 1.24 & 10.9 \\
GJ 402 & M4V & 1.34 & 1.55 & 1.57 & 8.4 \\
GJ 406 & M6V & 1.44 & 2.05 & 2.03 & 7.6 \\
GJ 569B & M8V & 1.31 & 1.68 & 1.29 & - \\
HR 7635 & M0III & 1.01 & 1.05 & 1.10 & 5.2 \\
HR 4202 & M1III & 1.05 & 1.10 & 1.16 & 3.5 \\
HR 7680 & M2.5III & 1.06 & 1.12 & 1.19 & 4.6 \\
HR 7645 & M4III & 1.11 & 1.16 & 1.36 & 2.1 \\
HR 6146 & M6III & 1.01 & 1.16 & 2.28 & 5.4 \\
\hline
\end{tabular}

We have used the spectral indices and the strength of the NaI doublet to classify the M-type stars in our sample. The stars that fall in category ii) are all dwarfs, except star 81 which could be a subgiant. These stars have the following Kun's (1992) numbers and spectral types: 1 (M0), 4 (M3.5), 12 (M3.5), 15 (M3), 32 (M1), 45 (M3.5), 49 (M1), 58 (M1.5), 60 (M4), 64 (M1), 73 (M3.5), 78 (M1), 81 (M4), 82 (M1), 84 (M4), 85 (M3), 88 (M3), 94 (M0). None of them show detectable $\mathrm{H} \alpha$ emission or Li I $\lambda 6708$ absorption.

\subsection{The Ho emission stars}

Stars with $\mathrm{H} \alpha$ emission are listed in Table 3 and displayed in Fig. 1. The spectral types were derived as described above. Luminosity class IV (subgiant) was assigned to stars showing weak $\mathrm{NaI}$ doublet, i.e. equivalent width (EW) less than $6 \AA$. Those with a strong Li absorption feature are accepted as T Tauri stars, and they are all lo- cated in the L134 cloud complex. In Fig. 2 we show the Li I region of several $\mathrm{H} \alpha$ emission stars.

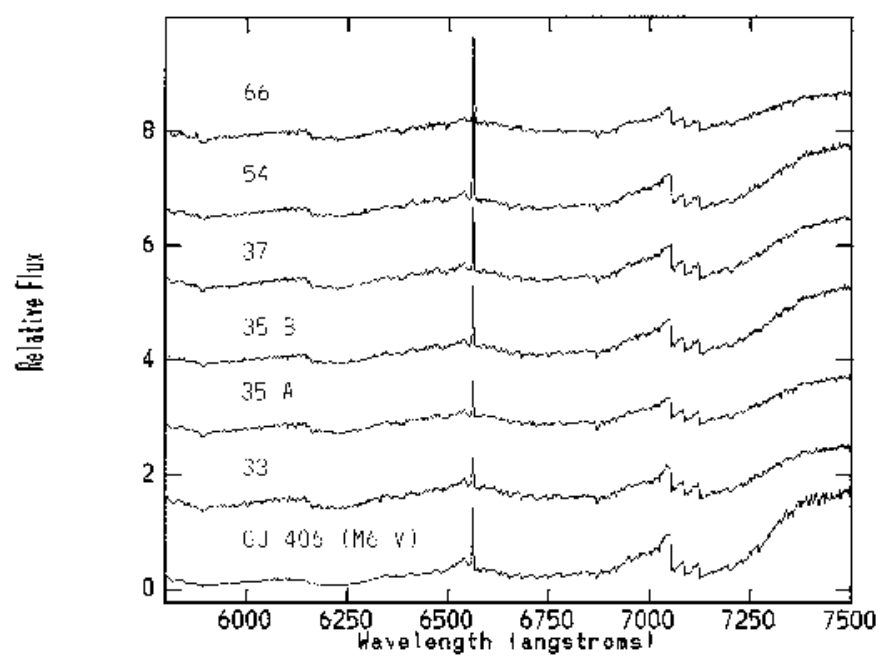

Fig. 1. Spectra of several $\mathrm{H} \alpha$ emission stars found in high latitude molecular clouds. All of them have late $\mathrm{M}$ spectral types (Table 3 ). The bottom star is a standard M6 dwarf. The spectra have been normalized at $\lambda 650 \mathrm{~nm}$, and shifted

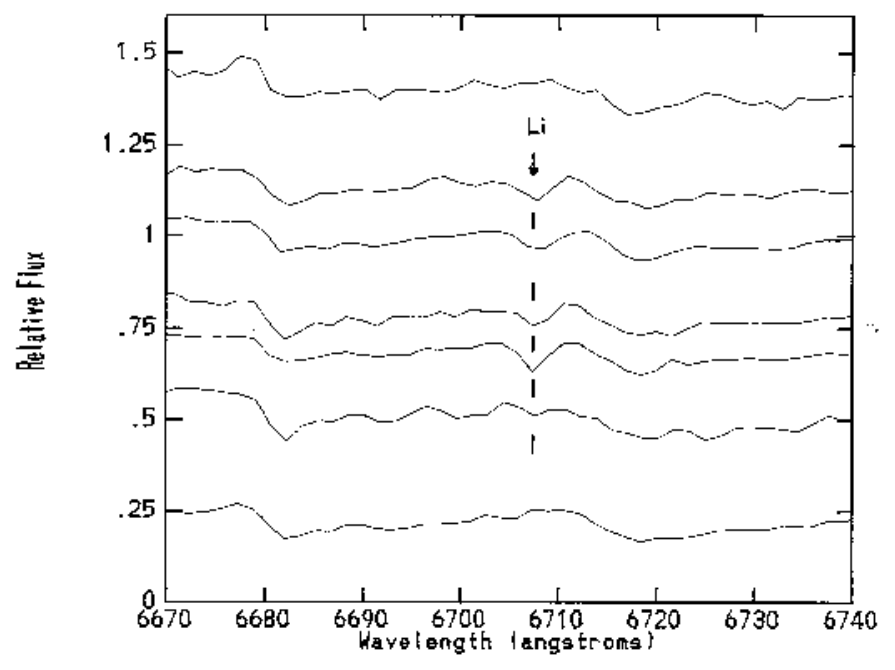

Fig. 2. Expanded spectra around the Li I line of the same stars as in Fig. 1. Note the Li I $\lambda 6708$ features in stars Nos. 54, 37 and the two visual components of 35

The EWs were derived as the mean value of several measurements using gaussian fitting and direct integration with several positions of the continuum level. The radial velocities were obtained from a gaussian fit to the position of $\mathrm{H} \alpha$ emission (see Martín et al. 1994b for more details). The $\mathrm{H} \alpha$ emission comes from the stellar chromosphere, and the centroid of the line should give the correct radial velocity for the star. 
Table 3. $\mathrm{H} \alpha$ emission stars

\begin{tabular}{llrrrl}
\hline $\begin{array}{l}\text { Kun's } \\
\text { number }\end{array}$ & Sp.T. & $\begin{array}{r}\mathrm{H}_{\alpha} \\
\AA\end{array}$ & $\begin{array}{r}\mathrm{Li} \mathrm{I} \\
\mathrm{m \AA}\end{array}$ & $\begin{array}{r}v_{\text {LSR }} \\
\mathrm{km} \mathrm{s}^{-1}\end{array}$ & Cloud \\
\hline 33 & M5 V & -2.9 & $\leq 260$ & 6 & MBM 32 \\
35 A & M4 V & -4.2 & 610 & -9 & MBM 33 \\
$35 \mathrm{~B}$ & M5 IV & -8.5 & 690 & -1 & MBM 33 \\
36 & M4 V & -4.0 & $<150$ & -29 & MBM 33 \\
37 & M4.5 IV & -8.3 & 610 & -24 & MBM 33 \\
52 & M4 V & -2.5 & $<110$ & -23 & MBM 37 \\
54 & M5.5 IV & -14.9 & 810 & -26 & MBM 37 \\
66 & M3.5 V & -12.3 & $<180$ & 0 & MBM 39 \\
83 & K7 V & -1.4 & $<70$ & variable & MBM 42 \\
86 & M3 V & -1.9 & $<120$ & -51 & MBM 50 \\
91 & M2 V & -3.1 & $<90$ & -16 & MBM 55 \\
\hline
\end{tabular}

Notes: Star number 35 is a visual binary with angular separation $\sim 7^{\prime \prime}$. Star number 83 is a double lined spectroscopic binary. The $1 \sigma$ error in the EWs is $\sim 5 \%$, and in the radial velocities $\pm 14 \mathrm{~km} \mathrm{~s}^{-1}$.

\section{Discussion}

The L134 molecular complex is the northernmost extension of the Scorpio - Ophiuchus star forming region. Its distance is $110 \mathrm{pc}$ (Franco 1989). A radio and infrared study of this cloud complex was made by Laureijs et al. (1995). Our T Tauri stars Nos. 35, 36 and 37 are located outside of the region studied by those authors. Their nearest cloud is L1780, a cometary globule recently studied by Tóth et al. (1995). The radial velocity of K35 is close to the velocity of L1780, but those of K36 and K37 differ from the cloud's value $\left(3.8 \mathrm{~km} \mathrm{~s}^{-1}\right.$, Laureijs et al. 1995). K52 and K54 are situated in a higher density part of the cloud complex. They can be associated with the dense core L183i according to Laureijs et al.'s CO observations, although their radial velocities are different.

Stars Nos. 35, 37 and 54 show strong Li I features. We note that few very late-M $\mathrm{T}$ Tauri stars with strong $\mathrm{Li}$ lines are known, and we plan to study the Li abundances in the near future using higher resolution spectroscopy and recent computations of the line formation (Pavlenko et al. 1995). Since $\mathrm{Li}$ is rapidly depleted in very low mass stars, albeit not in brown dwarfs (cf. Magazzù et al. 1993), these stars must be very young (age $\sim$ few $10^{6}$ years). No star formation was found earlier in the L134 molecular complex. Nevertheless, this is a peripheral part of a giant star forming region and not an isolated translucent cloud.

Star No. 83 is particularly interesting because it is projected on MBM 42. This cloud is the smallest clump of a high velocity molecular complex in Draco. Our spectrum did not have enough spectral resolution and $S / N$ ratio to confirm or not the presence of a weak Li I feature. During the refereeing process of the manuscript we took a higher resolution, higher $S / N$, spectrum with the INT on August 10, 1995. This spectrum shows double peaked $\mathrm{H} \alpha$ profile and double absorption lines. Thus, star No. 83 is a double-lined spectroscopic binary, its $\mathrm{H} \alpha$ emission can be explained by BY Dra type of activity, and it probably is not a PMS star.

Johnson (1986) argued that the $100 \mu \mathrm{m}$ IRAS point sources associated with the Draco molecular clouds are probably low mass protostars. On the contrary, Herbstmeier et al. (1993) found that the density of these clouds is low for star formation. Of course, the present low density of the MBM 42 clump only indicates that no further star formation is expected. Our results are consistent with Herbstmeier et al. because we do not find evidence for newly born stars (age $\leq 1 \mathrm{Myr}$ ).

It is remarkable that all four $\mathrm{T}$ Tauris found are of late $\mathrm{M}$ spectral type. Typically, $\mathrm{T}$ Tauri stars in well known star forming regions like Taurus-Auriga, have spectral types K7-M0. In fact, only very few T Tauris of late M spectral types are known. Although our sample is not statistically significant, it suggests that the physical conditions in high latitude clouds may favour the formation of very low mass stars.

\section{Summary}

We have performed intermediate resolution spectroscopic observations of 63 stars, which include all the best candidates for PMS stars among the 100 possible $\mathrm{H} \alpha$ emission objects identified by Kun 1992 in several regions of high latitude molecular clouds. The rate of successful confirmation of the $\mathrm{H} \alpha$ emission is low (only $\sim 20 \%$ ), but it shows that Kun's survey was able to identify weak $\mathrm{H} \alpha$ emission lines in faint stars near the plate magnitude limit $(V \sim 16)$. For instance, stars Nos. 83 and 86 have $\mathrm{H} \alpha$ emission equivalent widths less than $2 \AA$.

Four of Kun's candidates (Nos. 35 A, 35 B, 37 and 54) are bona fide $\mathrm{T}$ Tauri stars on the basis of their strong Li I absorption feature, $\mathrm{H} \alpha$ emission and late M spectral type. They are all located in the L134 molecular complex, and provide for the first time unequivocal evidence for star formation activity in this region.

The main result of this work is to demonstrate that Kun's survey was able to identify weak $\mathrm{H} \alpha$ emission lines in relatively faint stars. We note that most $\mathrm{T}$ Tauri stars known are brighter than the magnitude limit of Kun's plates. However, only 4 of Kun's candidates turned out to be T Tauri stars, and it is remarkable that they are all of very late spectral types. T Tauri stars usually have spectral types around $\mathrm{K} 7 / \mathrm{M} 0$, and they would have been easily detected because they are brighter. We also note that any classical $\mathrm{T}$ Tauri, with very strong emission lines, would not have escaped detection. Our results strongly constrain the presence of a population of $\mathrm{T}$ Tauri stars in high latitude clouds.

Acknowledgements. We thank Drs. L.G. Balázs and J.M. Rodríguez Espinosa for promoting this collaboration. We 
acknowledge INT service observations of six stars. We have made use of the La Palma ING data archive to retrieve some data. EM wishes to thank Nick Kylafis for his hospitality during his stay at FORTH (University of Crete). MK was partly supported by the Hungarian grant OTKA No. T4341.

\section{References}

Balázs L.G., Melikyan N.D., Melnikov S.Yu., Shevchenko V.S., 1987, IBVS No. 3099

Caillault J.-P., Magnani L., Fryer C., 1995, ApJ 441, 261

Franco G.A.P., 1989, A\&A 223, 313

Herbstmeier U., Heithausen A., Mebold U., 1993, A\&A 272, 514

Johnson H.M., 1986, ApJ 309, 321

Kirkpatrick J.D., Henry T.J., McCarthy D.W., 1991, ApJS 77, 417

Kun M., 1992, A\&AS 92, 875
Laureijs R.J., Fukui Y., Helou G., Mizuno A., Imaoka K., Clark F.O., 1995, ApJS (in press)

Magazzù A., Martín E.L., Rebolo R., 1993, ApJ 404, L17

Martín E.L., Rebolo R., Magazzù A., Pavlenko Y.V., 1994a, A\&A 282, 503

Martín E.L., Rebolo R., Magazzù A., 1994b, ApJ 436, 262

Martín E.L., Brandner W., 1995, A\&A 294, 744

Magnani L., Blitz L., Mundy L., 1985, ApJ 295, 402

Magnani L., Caillault J.-P., Armus L., 1990, ApJ 357, 602

Magnani L., Caillault J.-P., Buchalter A., Beichman C.A., 1995, ApJS 96, 159

Ogura K., Sato F., 1990, PASJ 42, 583

Pavlenko Ya.V., Rebolo R., Martín E.L., García López R. 1995, A\&A (in press)

Tóth L.V., Haikala L., Liljeström T., Mattila K., 1995, A\&A 295,755

Zapatero-Osorio M.R., Rebolo R., Martín E.L., García López R., 1995, A\&A (in press) 